\title{
Interpretar del neerlandés (L1) al español (L4) - Propuestas pedagógicas
}

\author{
Interpreting from Dutch (L1) into Spanish (L4) - \\ Pedagogical Proposals
}

\section{Hildegard VERMEIREN \\ Universiteit Gent \\ Hildegard.Vermeiren@ugent.be}

Recibido: 13-1-2016. Revisado: 28-10-2016. Aceptado: 1-11-2016.

Resumen: En el mundo existen muchos contextos donde los intérpretes tienen que trabajar tanto desde como hacia una lengua extranjera, que puede ser incluso una L4. Las facultades de interpretación se enfrentan a la tarea de preparar a sus alumnos para que salgan airosos de estos encargos. Sin embargo, día tras día, los profesores de interpretación son testigos de las incertidumbres, los errores y las estrategias de evitación de sus alumnos. Este artículo describe el método que se ha desarrollado en las clases de Neerlandés (L1)-Español (L4) del Máster en Interpretación de la Universidad de Gante. Sus ideas clave son: creación de redes semánticas y gramaticales y cambio de direccionalidad en los ejercicios para consolidar los conocimientos adquiridos durante la preparación y así ampliar la "disponibilidad» lingüística general. El método propuesto sirve para cualquier par de lenguas, aun teniendo en cuenta que cada par presenta sus propios problemas.

Palabras clave: Direccionalidad; interpretación bi-activa; fidelidad; disponibilidad lingüística; enseñanza.

Hildegard VERMEIREN Interpretar del neerlandés (L1) al español (L4) Propuestas pedagógicas 


\begin{abstract}
Worldwide, there are many different contexts where interpreters have to work from or into a language that is an L2, L3 or even an L4 to them. Interpreting schools therefore face the challenge of preparing their students to perform well in such situations. Every day, however, interpreting trainers observe their students' uncertainties, errors, interferences and avoidance strategies. This article presents a teaching method that has been developed in the Dutch (L1)-Spanish (L4) bi-active classes in the Master in Interpreting at Ghent University. Its key ideas are: creating semantic and grammatical networks and linguistic back-and-forth work to consolidate during training the knowledge acquired during the preparatory stage, and enhancing general linguistic «availability». The method we present can be used for any language pair, although each language pair needs to address its own problems.
\end{abstract}

Keywords: Directionality; bi-active interpreting; fidelity; linguistic availability; teaching.

Europa es un espacio multilingüe: la Unión Europea cuenta con 28 Estados miembros y 24 lenguas oficiales. Tanto a nivel institucional como a nivel del mercado, la interpretación entre las 24 lenguas es intensa. No solo se observa un volumen considerable de trabajo de interpretación entre el francés, inglés, alemán, español e italiano, consideradas lenguas centrales, sino también entre idiomas menos hablados y menos estudiados, como es el caso de la combinación neerlandés-español.

Las instituciones europeas son fieles a la filosofía de la Escuela de París (Seleskovitch y Lederer 1989, 134-135), según la cual un intérprete debe trabajar hacia su idioma materno (L1 o lengua A en la jerga de los intérpretes) si quiere producir un trabajo de calidad. En los mercados nacionales, sin embargo, los intérpretes suelen trabajar también por retour o en modo bi-activo, es decir tanto en su lengua materna como en una lengua extranjera (lengua B o C en la jerga de los intérpretes). En este artículo no entramos en el debate sobre la supremacía de la lengua materna. Hablaremos de los estudiantes flamencos que aprenden a interpretar entre el neerlandés ( $L 1)$ y el español (L4). Coincidimos con Kalina $(2005,37)$ en que la preocupación por la calidad sigue siendo la misma, con independencia de la direccionalidad.

Para ofrecer una imagen más clara de este par de lenguas no tan conocido, comentaremos primero los principales factores a tener en cuenta. Son de tipo externo, como el impacto de la estructura del Estado belga y su política lingüística, la política educativa de Flandes y las políticas de las universidades o facultades de interpretación. Los hay también internos, es decir, psicológicos, relacionados con el aprendizaje de las lenguas y la aptitud para interpretar. Juntos determinan el contexto educativo. Después profundizaremos en el par de lenguas neerlandés-español y los problemas a los que se enfrentan nuestros alumnos al interpretar hacia el español. Para concluir, propondremos un método para producir una mejor calidad en la interpretación hacia la L4. Esta investigación, por lo tanto, está orientada hacia la práctica (Seliger y Shohamy 1989, 17).

Hildegard VERMEIREN Interpretar del neerlandés (L1) al español (L4) Propuestas pedagógicas 


\section{LENGUAS, ENSEÑANZA DE LENGUAS Y FORMACIÓN DE INTÉRPRETES EN FLANDES}

Bélgica tiene tres idiomas oficiales: el neerlandés (hablado por el $60 \%$ de la población), el francés (39\%) y el alemán (1\%). Los territorios lingüísticos están fijados por la ley: el neerlandófono, el francófono, el germanófono y el territorio bilingüe de Bruselas-Capital. Desde 1989, Bélgica es un Estado Federal en el que la autoridad educativa reside en las regiones. Eso significa que la formación de intérpretes en zona neerlandófona (Flandes y Bruselas) y zona francófona (Valonia y Bruselas) no es necesariamente la misma. Y, de hecho, hay diferencias. Mientras que, desde la reforma de Bolonia, en Valonia existe un Máster en Interpretación de Conferencias de dos años, Flandes tiene un Máster en Interpretación de un año, seguido de un Posgrado en Interpretación de Conferencias, también de un año. En el máster flamenco, la interpretación es bi-activa en contextos bilaterales tales como ámbitos empresariales, servicios públicos, hospitales o tribunales. Ahora bien, a diferencia de las clases de francés, inglés o alemán, que pueden partir de un nivel alto al inicio de la carrera de traducción e interpretación, el español tiene que partir desde cero, porque en el contexto flamenco el español es una $L 4$ para la casi totalidad de los alumnos (Luján-Castro 2002, 29).

Con sus 25 millones de hablantes, el neerlandés es un idioma menor dentro de Europa. El inglés, el francés y el alemán están más presentes debido a su mayor peso demográfico (DG Communication 2012, 26). Además, son idiomas hablados en los países vecinos de Bélgica. Por eso reciben especial atención en la enseñanza belga. Como el italiano y el español son lenguas menos centrales, se estudian menos.

\section{VENTAJAS Y DESVENTAJAS DEL MULTILINGÜISMO}

Ser multilingüe puede suponer una ventaja, sobre todo cuando se trata de una lengua hipercentral (inglés) o central (francés, alemán, español) en la jerarquía (De Swaan 2001). Estos idiomas ofrecen muchas ventajas a la hora de usar las tecnologías de la información y comunicación, de viajar o de buscar trabajo en la misma Bélgica o en el extranjero.

El multilingüismo conlleva también ventajas cognitivas. Varios estudios han demostrado que existen diferencias entre la adquisición de la $L 2$ y la adquisición de más lenguas extranjeras. Al aprender una $L 3$, $L 4$, etcétera, el estudiante aprovecha una serie de competencias y conocimientos de los que carecía al aprender la L2. Eso abre el camino a lo que Hufeisen y Neuner $(2004,5)$ denominan «didáctica terciaria», es decir, una didáctica que tiene en cuenta estos conocimientos previos. El principal peligro son las interferencias. Green $(1998,73)$ sostiene que la persona multilingüe

Hildegard VERMEIREN Interpretar del neerlandés (L1) al español (L4) Propuestas pedagógicas 
es capaz de reprimir cualquier lengua que no sea la lengua meta mediante un control inhibitorio. Pero este control no es siempre perfecto.

Ortega (2009, 31-54) profundiza en las inevitables influencias entre idiomas que uno conoce. Plantea que las lenguas estudiadas con anterioridad pueden influir tanto negativa como positivamente en la nueva lengua. Cuando existe una afinidad tipológica, en muchos casos se puede recurrir a la transferencia. Sin embargo, en no pocas ocasiones el resultado es agramatical o no es idiomático. Guijarro-Fuentes (2012, 715 718) cree que estos errores ocurren porque determinados rasgos de la nueva lengua le resultan impenetrables al estudiante, o porque a lo mejor este reconoce los rasgos, pero no está acostumbrado a usarlos en la nueva combinación.

En Flandes, concretamente, hay que tener en cuenta que puede haber interferencias de otros idiomas. Un idioma que ocupa un lugar especial en Bélgica en este sentido es el francés (L2), porque se mantiene en una situación de contacto con el neerlandés a pesar de la frontera lingüística. La afinidad entre dos lenguas romances, como el francés y el español, es mayor que la que existe entre el español y una lengua germánica como el neerlandés. Por tanto, no es de extrañar que el francés a veces escape al control inhibitorio y asome cuando un flamenco aprende el español. Cuando un flamenco está hablando en neerlandés estándar, sabe que tiene que inhibir el uso de galicismos, pero estos pueden asomar inadvertidamente cuando habla en español (L4), y resultar acertados o no. Van der Sijs $(2009,196)$ menciona sucesivamente el francés (4648 palabras), el latín (1993 palabras) y el inglés (1863 palabras) como las lenguas de las que el neerlandés «importa» más vocablos. No es de extrañar, pues, que estas importaciones aparezcan cuando un alumno flamenco habla español. En una formación de intérpretes, donde los alumnos alternan continua y rápidamente entre dos idiomas, conviene aprovechar, por tanto, los efectos translingüísticos positivos y reducir los negativos.

\section{UN CONCEPTO PEDAGÓGICO PARA LA ENSEÑANZA DE LA L4}

\subsection{Competencias lingüísticas}

El objetivo de las facultades de traducción e interpretación es que sus estudiantes alcancen un nivel de competencia profesional al finalizar los estudios. La competencia más importante es la lingüística (Gile 2009, 8), tanto en la lengua materna como en las lenguas extranjeras. Otras competencias importantes del intérprete -que no comentaremos aquí- son la intercultural y la gestión de la interacción. El Marco Común Europeo de Referencia para las Lenguas (MCERL) aclara lo que la competencia lingüística significa para una L2 (Consejo de Europa 2002), con descriptores precisos para los niveles A-B-C. En términos del MCERL se trata en los estudios de interpretación de alcanzar un nivel C1 o C2 (usuario competente). ¿Cómo se puede lograr este nivel

Hildegard VERMEIREN Interpretar del neerlandés (L1) al español (L4) Propuestas pedagógicas 
en unos alumnos que no son bilingües en el sentido de que el español no es una lengua del ámbito familiar?

Para que sus alumnos alcancen el nivel C, las facultades de traducción e interpretación de Flandes basan su pedagogía en una doble metodología: la contrastiva, para la enseñanza de la gramática, así como la comunicativa, para la enseñanza de la lengua en general.

Enfocar las gramáticas neerlandesa y española desde el punto de vista contrastivo puede ser útil si uno no se ciñe a meras diferencias estructurales y se presta a razonar en términos de equivalencia. En concreto, para el estudio del español, los neerlandófonos disponen de la gramática española de De Bruyne y Behiels (2013). Aunque ésta no se define como comparativa, ni siquiera contrastiva, esclarece un importante número de similitudes y diferencias entre el neerlandés y el español. A menudo va incluso más allá de las diferencias estructurales y se refiere a la dificultad de encontrar un equivalente o una traducción. La obra también expone las asimetrías conceptuales entre el neerlandés y el español. Los autores, de hecho, se refieren una veintena de veces a la similitud entre el español y el francés, sobre todo en los capítulos sobre sintaxis española. Tales observaciones son relevantes solo para lectores que también conozcan el francés, como suele ser el caso en Bélgica, pero mucho menos en los Países Bajos. Por tanto, esta gramática es una obra de referencia útil para los traductores e intérpretes flamencos que trabajan hacia el español.

Desde los años 90, el conocimiento de la gramática de la lengua extranjera ya no se ve como un fin en sí, sino como un componente de lo que es el objetivo clave del MCERL: la capacidad de comunicar en un idioma extranjero. En la carrera de traducción e interpretación, los alumnos recorren a lo largo de los años de Grado los niveles A y B del MCERL para alcanzar al final de sus estudios el nivel C (usuario competente). El MCERL (Consejo de Europa 2002, 85-86) explora brevemente las actividades de interpretación y traducción, a las que llama «mediación». El documento se refiere a los pocos recursos disponibles para procesar la información y establecer el significado equivalente; se refiere a los «malabarismos» del intérprete, las técnicas para eludir incertidumbres y para evitar interrupciones. El MCERL no explicita qué nivel de lengua hay que tener para realizar esta actividad, como tampoco indica si la mediación oral hacia una lengua extranjera plantea desafíos diferentes. Señala que los usuarios (profesores) pueden determinar qué se puede exigir al alumno.

Desde la difusión del MCERL, interpretar se ve como una acción comunicativa. Por tanto, los descriptores de la competencia lingüística de los niveles C1 y C2 son relevantes para la recepción y la producción en lengua extranjera. Interpretar conlleva, además de aspectos gramaticales y léxicos, aspectos sociolingüísticos y pragmáticos, así como estrategias. De hecho, en una situación de mediación no solo se trata de comunicar en un lenguaje correcto y fluido, sino que importa también la fidelidad al mensaje. Para ser fiel al mensaje, las «estrategias» usadas en la fase de producción

Hildegard VERMEIREN Interpretar del neerlandés (L1) al español (L4) Propuestas pedagógicas 
cobran una importancia particular. El MCERL afirma, en el nivel C, que el usuario tiene que saber suplir lagunas, tanto estructurales como léxicas. Pero eso no justifica que al interpretar se haga uso constantemente de estrategias de evitación (Ortega 2009, 39-41) porque, al eludir formulaciones precisas, el resultado será una traducción de mala calidad.

\section{2. ¿Qué es la interpretación bi-activa?}

En estas páginas abordamos la interpretación en la lengua extranjera, algo corriente en una situación de interpretación bi-activa. Nos limitamos a la bi-activa consecutiva, sin adentrarnos en otras modalidades como la simultánea susurrada o la traducción a vista. Para saber cuáles son exactamente los desafíos cognitivos planteados por la interpretación en una L4 tenemos que definir primero qué es la interpretación consecutiva.

Interpretar es un comportamiento humano que se produce en situaciones que el MCERL define como «mediación». Cuando en el contexto de una comunicación multilingüe se levanta una barrera lingüística entre los interlocutores, esta barrera se puede traspasar haciendo uso de un «mediador» que reformula de forma inmediata el mensaje de un interlocutor en la lengua del otro. A esta persona se la llama «intérprete» y lo que hace es «interpretar». El intérprete explica a la segunda persona lo que acaba de decir la primera (Jones 1998, 3). Ello implica hacer uso de los canales sensorial y verbal.

Gile (2009, 101-127) comenta el tradicional modelo secuencial en dos pasos (comprensión/reformulación) para la traducción/interpretación en general. Encontramos el mismo modelo en dos fases en el perfil profesional para intérpretes sociales que se ha desarrollado en Flandes, y que habla de recepción (en neerlandés: «verwerken») y reproducción (en neerlandés: «reproductie») (SERV 2008). En ambos casos se insiste en que se trata de establecer un significado equivalente entre las dos fases.

El paso 1 consiste en la recepción y el análisis del mensaje. El paso 2 consiste en la reproducción fiel del mismo mensaje en otro idioma.

\section{PASO 1: RECEPCIÓN Y ANÁLISIS}

Cuando el primer interlocutor toma la palabra en la $L 1 \circ L 2, L 3$, L4, etc. del intérprete, este escucha con atención, no para reaccionar él mismo a lo que oye, sino con intención de reproducir a continuación el mensaje oralmente en otro idioma para otro interlocutor. Los descriptores específicos de la técnica usada son los siguientes: al intérprete le basta oír una vez lo que dice el interlocutor para captarlo todo y situarlo en un contexto más amplio; capta el mensaje en vez de fijarse solo en las palabras; almacena el mensaje en su memoria de trabajo - haciendo uso eventualmente de notas - para reproducir luego este mismo mensaje para el otro

Hildegard VERMEIREN Interpretar del neerlandés (L1) al español (L4) Propuestas pedagógicas 
interlocutor. Las notas (tomadas en lengua origen o parcialmente en lengua meta, a menudo para apuntar nombres y cifras) adoptan la forma de palabras clave y conectores reflejados en palabras, abreviaciones o símbolos. Todo se desarrolla en un contexto de interacción: el intérprete muestra que está escuchando activamente, y si no ha oído o entendido bien al orador puede pedirle que repita o aclare lo dicho, o puede interrumpirlo para interpretar. La primera fase implica una actividad sensorial (escuchar) y cognitiva (comprender, analizar) considerable. Generalmente, en una situación de consecutiva la fase de recepción dura unos minutos.

\section{PASO 2: PRODUCCIÓN}

En cuanto el orador se para, el intérprete toma la palabra. Los descriptores de una técnica de interpretación efectiva son: el intérprete activa la lengua meta (extranjera o nativa) y arranca pocos segundos después de que el orador haya dejado de hablar, articula claramente y adopta una entonación adecuada; se vale de las notas y de lo que ha almacenado en la memoria de trabajo para reformular el mensaje en la lengua meta; este cambio de lengua ya se puede observar en las notas; la reproducción en la lengua meta es fluida y continua, adecuada desde los puntos de vista sociolingüístico y pragmático, y ocurre bajo un monitoreo constante en el que los elementos nuevos enlazan con los anteriores; la autocorrección se limita al mínimo; en esta fase el intérprete ya no se dirige al orador para pedir clarificaciones o repeticiones, y tampoco se deja interrumpir antes de reproducir la totalidad del mensaje. Algo de suma importancia en esta fase es el descriptor «fidelidad»: el contenido del mensaje no debe alterarse en la transferencia a la lengua meta, lo que incluye por ejemplo traducir también insultos o lenguaje soez. La fase de producción implica una actividad sensorial importante (leer las notas), unida a una actividad cognitiva (hacer comprensible el mensaje para otros) y fisiológica (hablar para que otros puedan captar el mensaje). Esta fase generalmente tiene la misma duración que la fase de recepción o es ligeramente más breve.

Ahora bien, en un contexto bi-activo la interpretación hacia la lengua extranjera supone un esfuerzo adicional:

- El cambio de dirección constante causa cansancio cognitivo - sobre todo durante la fase de producción - y puede hacer que el intérprete se equivoque de lengua o que su atención disminuya.

- Al cambiar constantemente de idioma, el intérprete se expone a interferencias de una lengua en la otra, especialmente de la más fuerte en la más débil. Por eso precisamente es importante que el intérprete alcance el nivel C de usuario competente. Solo así puede ejercer control sobre su producción y evitar interferencias.

Hildegard VERMEIREN Interpretar del neerlandés (L1) al español (L4) Propuestas pedagógicas 
- En el plano del repertorio lingüístico la producción en la L4 puede verse afectada por interferencias de otros idiomas que conoce el intérprete, ya sea en sentido positivo o negativo. De Groot $(2011,337)$ señala que el intérprete aprovecha las similitudes entre idiomas para reducir el esfuerzo cognitivo.

- Los alumnos acuden a menudo a estrategias de evitación debido a las lagunas en sus conocimientos de la lengua extranjera.

Aun así, la interpretación bi-activa presenta un aspecto que permite que el intérprete se relaje. De hecho, puede aprovechar el diálogo en las dos lenguas para reutilizar las formulaciones de los interlocutores al dirigirse a su vez a ellos (Collados Aís y Fernández Sánchez 2001, 65), lo que le permite ampliar su repertorio lingüístico durante el encargo mismo.

Hay otro aspecto más que incluso un documento tan general como el MCERL menciona para cualquier actividad de «mediación»: las incertidumbres (Consejo de Europa 2002, 86). En su modelo secuencial Gile $(2009,103)$ insiste en que durante la fase de escucha y análisis el intérprete formula una hipótesis sobre el mensaje que está oyendo en la lengua fuente, que en este caso es la lengua extranjera. Hatim $(1997,3)$ lo llama la «heurística» del intérprete. Ahora bien, ni Gile ni Hatim mencionan una hipótesis similar durante la fase de reformulación, donde en realidad el intérprete también se siente inseguro, esta vez en cuanto a la formulación más adecuada. Padilla (2005, 33-35), en cambio, afirma que para un intérprete que trabaja hacia una lengua extranjera puede resultar difícil tomar decisiones lingüísticas. Las decisiones que debe tomar un intérprete durante la fase de producción en la L2, L3, L4, etcétera, suelen ir acompañadas de suposiciones e hipótesis, tanto más porque el intérprete dispone de poco tiempo para encontrar una solución. El objetivo del enfoque pedagógico que preconizamos es que las suposiciones e hipótesis tanto léxicas como gramaticales del intérprete que trabaja hacia la lengua extranjera sean lo más correctas e idiomáticas posibles, y que por tanto se usen pocas estrategias de evitación, que desembocan en traducciones sin calidad.

\subsection{El par de lenguas neerlandés y el español: afinidad}

Pöchhacker $(2004,133)$ señala que, desde sus inicios, los estudios de la interpretación han prestado atención a las diferencias estructurales entre pares de lenguas y las estrategias para sobrellevarlas. Pero Pöchhacker una vez más se refiere a la interpretación simultánea hacia la lengua materna, y no a la consecutiva hacia la lengua extranjera. En una situación de consecutiva, el intérprete dispone de más tiempo para encontrar soluciones, y puede solucionar más al nivel del texto, mientras que en la simultánea hay que encontrar soluciones al nivel de la frase. Gile señala que hay pares

Hildegard VERMEIREN Interpretar del neerlandés (L1) al español (L4) Propuestas pedagógicas 
de lenguas que plantean más problemas que otras, por la gran distancia que existe entre ellas, por ejemplo entre el chino y el inglés (Gile 2005, 17). Lo que Gile señala es también relevante para los intérpretes en la modalidad de consecutiva tanto hacia la $L 1$ como la L2, L3, etcétera: interpretar en determinada dirección puede ser más fácil o más difícil según la afinidad o distancia entre la sintaxis, el léxico común, el isomorfismo entre campos léxicos y las costumbres culturales de los oradores (por ejemplo, usar con frecuencia giros idiomáticos, citas). Gile señala, además, la existencia de lo que él llama el «Escort Effect» (Gile 2009, 231): al interpretar entre lenguas más o menos afines, uno a veces puede aprovecharse de las similitudes, pero otras veces cae en trampas, las llamadas «interferencias lingüísticas».

Estas similitudes e interferencias lingüísticas se dan entre el neerlandés y el español. Ambas lenguas son indogermánicas occidentales, de modo que no es sorprendente que existan similitudes entre ellas. Además, el francés (L2) actúa como puente: su conocimiento ayuda a un neerlandófono a comprender y a hablar el español (L4).

\subsection{Desafíos lingüísticos en la interpretación del neerlandés al español}

Al inicio del Máster en Interpretación, los alumnos en Flandes todavía no han alcanzado el nivel C del MCERL en español (L4). Presentan lagunas, tanto en el conocimiento de la gramática, el léxico, la pragmática y los aspectos sociolingüísticos, como en el manejo de la comunicación/interacción. Aunque el conocimiento de otras lenguas (especialmente el francés) ayuda, también puede ser una fuente de problemas, sobre todo en lo que respecta a los «falsos amigos». En esta fase, a los alumnos aún les supone un gran esfuerzo traducir oralmente hacia la lengua extranjera, ya que corren el riesgo permanente de cometer errores o tener que recurrir a laboriosas estrategias de evitación.

Por eso, en las líneas que siguen queremos recopilar primero una serie de dificultades que se plantean en la producción en L4 español por neerlandófonos. No nos interesan ahora los problemas de comprensión y de traducción del español (L4) al neerlandés (L1), que se abordan en Linn y Slager (2007), aunque es cierto que muchas veces plantean el mismo problema que en esta recopilación pero al revés. Tampoco podemos profundizar aquí en la influencia del francés u otras lenguas en la producción en español, lo cual exigiría un estudio longitudinal con una considerable recogida de datos orales, seguidos de su transcripción y análisis. Lo que nos interesa no es el análisis de errores, sino el tipo de problemas en los que los alumnos tienen que invertir energía en lugar de concentrarse en la mera interpretación.

Queremos presentar la metodología desarrollada a lo largo de una década de clases de interpretación bilateral neerlandés-español en el Máster en Interpretación de Gante. El objetivo es que los alumnos venzan dificultades lingüísticas y mejoren su nivel

Hildegard VERMEIREN Interpretar del neerlandés (L1) al español (L4) Propuestas pedagógicas 
de español. Deben lograr una producción oral de calidad, es decir, lingüísticamente correcta y que además sea la reproducción fiel de lo que ha dicho otra persona.

\subsubsection{Pronunciación}

En el momento actual, los intérpretes están muy influidos por la grafía. Han estudiado la lengua extranjera por medio de manuales, diccionarios y textos, aunque no puede decirse que los fragmentos de audio y vídeo hayan faltado. El neerlandés y el español tienen más o menos la misma serie de grafemas (alfabeto), pero los fonemas no son siempre idénticos. Ello hace que los alumnos tiendan a pronunciar algunas palabras españolas «a la neerlandesa». En español, la pronunciación es ligeramente diferente en el caso de los grafemas b, c, d, g, j, (II), q, r, (rr), v, x, z. Sobre todo la pronunciación de los grafemas $\mathrm{r} / \mathrm{rr}, \mathrm{g} / \mathrm{j}$ y $\mathrm{c} / \mathrm{z}$ suponen una dificultad que no todos los alumnos han superado después de tres años de Grado. Palabras como 'Miguel', 'queso', 'guerra' se pronuncian a menudo como si tuvieran un diptongo 'ue'. El diptongo 'ue' español a veces se pierde como cuando 'cuestión' se transforma en 'question' bajo la influencia del francés. El mismo diptongo 'eu’ en 'Europa' o ‘euro' a menudo se pronuncia como 'oi', a saber, 'Oiropa' y 'oiro', como en alemán. 'Patio' (con el sonido 'ts') y 'casa' (con ' $z$ ' sonora) se pronuncian a menudo como si fueran palabras italianas. Las vocales plantean menos dudas que los diptongos, aunque a veces ocurre que los alumnos pronuncian la 'e' española como una 'e' átona como en neerlandés o francés. Otro problema es la simple pronunciación del nombre de la letra cuando hay que deletrear o usar siglas. A veces surgen dudas como lo demuestra la siguiente frase en la que una alumna deletrea mal las siglas 'UZA' (del hospital universitario de Amberes), cuando dice «La u zete a... uve zete a... es decir, el hospital universitario de Amberes».

En ambos idiomas, el acento cae en las vocales. En neerlandés, el acento se oye, pero no se escribe. En español, el acento se oye y es a veces ortográfico, según unas reglas que los alumnos flamencos a veces tardan en entender. Algunos errores de acentuación pueden explicarse por la influencia del neerlandés, por ejemplo en 'Se trata de un parasito' (correcto: parásito), y del francés, por ejemplo en 'etceterá' (correcto: etcétera).

\subsubsection{Léxico}

Aunque gran parte del léxico no coincide, el neerlandés y el español tienen numerosos cognados. Hay un riesgo permanente de calcos del neerlandés, como por ejemplo hablar de 'toneladas' cuando se trata de 'barriles' o de 'doctores' cuando se trata de 'médicos'. Los galicismos usuales en el neerlandés de Bélgica pueden

Hildegard VERMEIREN Interpretar del neerlandés (L1) al español (L4) Propuestas pedagógicas 
hispanizarse fácilmente. Del mismo modo que los sustantivos, los adjetivos a veces se pueden copiar literalmente del neerlandés (o del francés), sobre todo cuando tienen raíces latinas. Pero eso puede inducir a error, como por ejemplo en 'Es una persona sadística' (correcto: sádica) o en 'amusante' (divertido). Otros ejemplos de préstamos malogrados del francés son: 'apartenecen' (pertenecen), 'pasarse' (pasar), 'explosar' (hacer explosión) 'reúnen bicicletas' (arman bicicletas), 'coleccionar pruebas' (reunir pruebas). Otro problema es la formación de palabras. En general el español es más analítico y el neerlandés más sintético cuando se trata de compuestos. Mientras que el neerlandés puede hacer compuestos de forma bastante libre, en español por lo general hay que usar un grupo compuesto por palabra+de+palabra. Eso significa que, en español, el intérprete o bien necesita más palabras que en neerlandés, o bien tiene que buscar una palabra totalmente diferente. Hay diferencias conceptuales entre los verbos en neerlandés y en español, en cuyo caso los alumnos no siempre saben bien qué elegir. Los casos más conocidos son: 'haber'/'tener' (neerlandés: 'hebben'), 'ser'/'estar' (neerlandés: 'zijn'), 'llevar'/'traer' (neerlandés: 'brengen'), 'pedir'/'preguntar' (neerlandés: 'vragen'), 'morir'/'morirse' (neerlandés: 'sterven'). No sorprende, por tanto, que los alumnos construyan frases como: '¿Su partido ha una opinión sobre el asunto' (tiene), 'Es importante ser informado' (estar), 'X se ha muerto en un accidente de circulación' (ha muerto).

\subsubsection{Gramática}

Morfología. El español tiene una morfología nominal y verbal más analítica que el neerlandés (por ejemplo, en las formas nominales hace mención continua al género y al número, lo que el neerlandés no hace). A un neerlandófono, estas categorías no le resultan extrañas, pero lo que sí supone un esfuerzo en la producción es la concordancia. Por consiguiente, los alumnos se equivocan a menudo de género como en 'el África negro' (negra), 'buenas días' (buenos días); de persona, como en 'ya mencionó que...' (mencioné); y de número, como en 'estos mensajes están dirigido a...' (dirigidos).

Numerales. Ambas lenguas comparten el sistema numérico decimal. Aun así, los alumnos tropiezan con la formación de los números cardinales complejos, especialmente si contienen las cifras 1 y 100, y si hay múltiplos de 100-1000. Les cuesta transformar 'miljarden' del neerlandés (y 'milliards' del francés) en 'mil millones'; piensan a menudo que (el falso amigo) 'un millar' es la traducción correcta. Algo que complica aún más la situación es el monitoreo de la flexión nominal y poner algunas de las cifras en femenino, por ejemplo. Otro problema es la derivación de los numerales ordinales: en neerlandés se hace con un simple afijo ('-ste' o '-de' después del numeral cardinal), pero en español es bastante complicado, y por eso se evita. Las apócopes

Hildegard VERMEIREN Interpretar del neerlandés (L1) al español (L4) Propuestas pedagógicas 
de 'uno', 'ciento', 'primero', 'tercero', exigen que los alumnos presten atención a las palabras adyacentes. Dicen por ejemplo algo como 'un millón ciento mil bicicletas' (correcto: cien mil). Los alumnos no suelen conocer los numerales fraccionarios y multiplicativos, como tampoco saben muy bien cómo formular operaciones aritméticas o incluso expresar porcentajes. No es sorprendente que digan 'un por ciento' (calco del neerlandés o del francés) cuando debe ser 'el uno por ciento'.

Pronombres y artículos. Los dos idiomas tienen pronombres personales, posesivos, demostrativos, indefinidos, reflexivos, recíprocos, exclamativos e interrogativos, como también artículos determinados e indeterminados. Sin embargo, puede haber diferencias en el uso (por ejemplo, en las formas tónicas o átonas que existen en ambos idiomas). Los neerlandófonos pueden preguntarse qué pasa con el adverbio pronominal anafórico neerlandés 'er' ('de ello') en español, o en qué momento el sujeto puede ser tácito o debe expresarse, por ejemplo en 'Yo voy a explicar qué se puede hacer contra...' (voy a explicar). Les cuesta evitar el leísmo-loísmo-laísmo, así como usar la correferencia con pronombres personales (objeto directo/indirecto), algo que el neerlandés no conoce. Otro fenómeno nuevo para ellos son los pronombres personales, tanto los proclíticos como los enclíticos con los verbos (lo que complica también la acentuación), o la relación entre 'se'/‘uno' en 'Se podría preguntarse la importancia de...' (uno podría preguntarse). El uso de los pronombres personales es más complejo que en neerlandés. Un error en su uso no implica necesariamente que la frase sea incomprensible, pero sí afecta a la precisión, como en 'Señoras y señores, le he hablado de...' (les he hablado). No es fácil para un neerlandófono usar los pronombres de manera idiomática en español. Los dos idiomas conocen tres géneros, pero estos no se expresan de la misma manera y el pronombre neutro no cumple las mismas funciones. La presencia o ausencia obligatoria de artículos no resulta clara. A nuestros alumnos les puede parecer correcto formular frases como 'Esquizofrenia es una enfermedad mental...' (La esquizofrenia) o 'Voy a hablar de dengue' (del dengue), porque en neerlandés el sustantivo genérico suele aparecer sin artículo. 0 '23 por ciento' (el 23 por ciento). Y al revés: 'No habrá una prima para los que...' (no habrá prima), con un artículo que es superfluo en español, cuando en neerlandés hay un artículo indeterminado negativo (geen). Puede haber diferencias conceptuales, como es el caso entre los pronombres demostrativos en neerlandés (dos: deze-die) y español (tres: este-ese-aquel), lo cual provoca inevitablemente desaciertos.

Preposiciones. Ambas lenguas usan preposiciones, en grupos preposicionales (preposición + sustantivo) y como prefijos preposicionales (por ejemplo, subrayar/ onderstrepen). La influencia del francés se nota en la aparición a veces superflua de la preposición 'de', por ejemplo en 'Será un placer de responder' (sobra 'de') o la aparición errónea de 'a' en 'Pensemos a Barcelona, Valencia' (en). El mayor problema

Hildegard VERMEIREN Interpretar del neerlandés (L1) al español (L4) Propuestas pedagógicas 
para los neerlandófonos es el uso de 'a acusativo' para personas. Guijarro-Fuentes $(2012,718)$ sostiene que puede que un estudiante conozca desde su propia lengua los rasgos que determinan la aparición de una u otra preposición, pero que no sea capaz de aplicarlos en el contexto de una frase concreta. Las preposiciones 'por'/'para' plantean un problema similar, por ejemplo en 'Es demasiado por esta persona' (para), 'Le quiero agradecer por su atención' (sobra 'por').

Negación. Los pronombres indefinidos, adjetivos, adverbios y conjunciones negativos no constituyen un problema en sí. La mayor novedad para un neerlandófono reside en la sintaxis española, que exige la alternancia negativa, algo que en neerlandés es agramatical. Por ejemplo: 'Nunca alguien ha visto a Dios' en vez de 'Nadie ha visto nunca a Dios'.

Sintaxis. Existe una gran similitud estructural entre ambas lenguas, pero cada una elige sus propias soluciones y las explota en mayor o menor grado. En cuanto al orden de los elementos, tanto el sintagma nominal como el sintagma verbal plantean problemas. Hay una diferencia notable en la posición del adjetivo en el grupo nominal. En neerlandés, el adjetivo siempre precede al sustantivo; en español, algunos adjetivos se anteponen y otros se posponen. A un neerlandófono no siempre le resulta fácil captar los rasgos semánticos que determinan dónde se pone el adjetivo en español. Ello es importante, porque el mensaje puede cambiar según la posición. Los alumnos tienden a colocar los adjetivos detrás del sustantivo, por ejemplo en 'Otra noticia mala es...' (correcto: otra mala noticia es...). Los intérpretes sin duda tienen la ventaja de que en el lenguaje hablado en general hay menos concurrencia de adjetivos, o que se acepta que alguno de los adjetivos desaparezca o se transforme en una oración independiente. En cuanto al sintagma verbal, ambas lenguas hacen uso de la estructura básica transitiva SVO y variantes, pero los alumnos no saben qué orden (o inversión en VSO) es posible en qué circunstancias. En general, no saben muy bien qué estructuras se apartan de la SVO, como se ve por ejemplo en '¿Qué ustedes tienen que hacer?'. Tanto el neerlandés como el español usan las formas no personales del verbo (participio, gerundio, infinitivo), pero el español las usa de manera más habitual que el neerlandés, sobre todo el gerundio. Las perífrasis verbales se asimilan sin mayores problemas, pero los demás usos suelen plantear dificultades. Uno de los mayores problemas entre el neerlandés y el español es la relación entre oraciones activas, pasivas, impersonales o pasivas reflejas. Los alumnos tienden a abusar de la pasiva con 'ser' (que copia la tradicional pasiva con 'worden' del neerlandés) y a infrautilizar la pasiva refleja (que, sin embargo, es el mejor equivalente para las construcciones con 'er + worden' en neerlandés). Existe una importante asimetría conceptual entre los tiempos, aspectos y modos verbales de ambos idiomas. Los tiempos verbales del español no coinciden con los del neerlandés (que tiene menos tiempos, como tampoco tiene subjuntivo). En

Hildegard VERMEIREN Interpretar del neerlandés (L1) al español (L4) Propuestas pedagógicas 
consecuencia, los estudiantes tienen que manejar unas categorías gramaticales que les resultan extrañas. A este respecto puede ser útil el conocimiento del francés, ya que tiene un sistema similar al español.

\subsubsection{Pragmática}

Para la traducción de los actos del habla no valen las traducciones literales del neerlandés al español. Solo caben expresiones equivalentes, que los alumnos no siempre conocen. Las fórmulas de cortesía también son específicas para cada lengua. En español suponen el conocimiento del subjuntivo. Al alumno flamenco le cuesta usar las fórmulas del tratamiento de cortesía; prefiere tutear al interlocutor, que es lo que enseñan los métodos comunicativos. El resultado son unos tratamientos híbridos como en la frase 'Señoras y señores, os voy a hablar...' (correcto: les).

Si consideramos todos los idiomas del mundo, podemos decir que hay similitudes considerables entre el neerlandés y el español. A pesar de sus diferencias comparten grafemas/fonemas y rasgos gramaticales comunes, así como muchos cognados (a menudo apoyados por el contacto con el francés). Pero a pesar de tener las mismas categorías gramaticales, estas no se usan de la misma manera, y a veces de forma infinitamente más detallada, como es el caso del sistema pronominal español. Eso hace que los estudiantes de interpretación en L4 español se tengan que enfrentar a varios desafíos. Las lenguas estudiadas anteriormente pueden ser una ayuda, y al mismo tiempo una trampa, para hablar la L4 español.

El intérprete se enfrenta a todas estas similitudes y diferencias en tiempo real, y sin poder someterlas a mucha reflexión. No puede decir cualquier cosa, sino que debe ser fiel al mensaje de otra persona. Si quiere hacer un trabajo profesional, necesita poder activar sus conocimientos con rapidez y sin fallos. Necesita disponer de un amplio abanico de recursos lingüísticos (léxicos y gramaticales). Por tanto, debemos desarrollar en nuestros estudiantes la agilidad mental necesaria para que durante un ejercicio de interpretación no pierdan tiempo (ni gasten energía mental) buscando equivalencias o monitoreando construcciones gramaticales, para que no cometan errores que den lugar a malentendidos, para que no se autocorrijan constantemente, ni para que terminen frustrándose, ni ellos mismos ni sus clientes.

\section{APRENDER A INTERPRETAR EN UNA L4: APRENDIZAJE INTENCIONAL EN CONTEXTO}

Hemos repasado los desafíos lingüísticos planteados por la interpretación del neerlandés en la $L 4$ español señalando de paso las ventajas e inconvenientes del

Hildegard VERMEIREN Interpretar del neerlandés (L1) al español (L4) Propuestas pedagógicas 
conocimiento de otras lenguas como el francés. La pregunta es cómo podemos conseguir que nuestros alumnos tengan la mayor disponibilidad léxica y gramatical posible en la L4 para vencer el mayor número posible de obstáculos, y lograr así una producción correcta, fluida y fiel. Desde luego, en el Máster en Interpretación no hay tiempo para volver a explicar toda la gramática y el vocabulario de los cursos de Grado. Como mucho, algunos temas se pueden repasar o ampliar un poco. Tampoco nos parece aconsejable que los profesores carguen toda la responsabilidad sobre los alumnos (por ejemplo, animándolos a leer en español, escuchar grabaciones, buscar amigos hispanohablantes o contratar clases particulares) y se centren únicamente en las técnicas de interpretación durante las clases.

\subsection{Disponibilidad léxica y gramatical}

Algunos investigadores y profesores de interpretación han esbozado metodologías para abordar el fortalecimiento de la lengua extranjera en un contexto de interpretación hacia la L2, L3, etcétera. Gile (2009, 220-231) dedica mucha atención a la disponibilidad léxica de los intérpretes en general, señalando que un léxico pobre lleva a un resultado insatisfactorio o poco satisfactorio para los clientes. Según él, no es difícil mejorar los resultados: explica que los «elementos constituyentes» (unidades léxicas) se vuelven más disponibles según crece su frecuencia de uso (activo) en diferentes situaciones, y de preferencia en un contexto, no en una simple lista de palabras. Gile se refiere, además, a la «adquisición de conocimientos ad hoc», es decir, el esfuerzo que realiza el intérprete cuando prepara un encargo y que supone una relación muy estrecha entre el contexto (el contenido o tema del encargo) y su lenguaje (Gile 2009, 129).

La enseñanza de la interpretación en la L2, L3, etcétera, que propone Donovan (2005, 163 y ss.) se enmarca en esta misma línea. Según la autora, hace falta un esfuerzo extraordinario por parte de los profesores y alumnos para fortalecer tanto la recepción como la producción, así como para ampliar el conocimiento de una gran variedad de temas (background knowledge) y su terminología. Subraya, además, que la propia metodología tiene como objetivo familiarizar a los alumnos con el modo de preparar un encargo profesional a corto plazo.

Setton y Drawant (2016a, 223 y ss.) vuelven sobre el tema de la interpretación en la $L 2, L 3, L 4 \ldots$, insistiendo una vez más en la importancia de la disponibilidad léxica y la falta de fluidez que se manifiesta en formulaciones incoherentes, omisiones, incapacidad de encontrar soluciones, autocorrecciones molestas, oraciones sin terminar, interferencias de otro idiomas, mezcla de registros y otras incongruencias, así como en la falta de control gramatical. Asimismo, los autores afirman que el fortalecimiento lingüístico debe ir acompañado de la ampliación de los conocimientos generales de los estudiantes en la fase de preparación del encargo, es decir, cuando

Hildegard VERMEIREN Interpretar del neerlandés (L1) al español (L4) Propuestas pedagógicas 
estudian el tema, elaboran glosarios y memorizan (priming) los términos y expresiones (Setton y Drawant 2016a, 216). Sin embargo, no indican cómo implementar todas estas ideas en clase en el marco de un curso o una asignatura.

En las líneas que siguen, detallamos una propuesta que parte de las pautas señaladas por los investigadores y los intérpretes profesionales. Nos inspiramos en cómo afrontan los intérpretes profesionales los desafíos de su profesión día tras día, encargo tras encargo. Es evidente que para mejorar la producción en la L4 es necesario pensar tanto en la adquisición de conocimiento temático como en los aspectos lingüísticos (tanto de la L1 como de la L4). Además, creemos que no conviene obligar a los alumnos a buscar de forma independiente soluciones para practicar la L4. Al contrario, tenemos que encontrar una solución que les permita fortalecer sus conocimientos gracias a nuestras clases, de tal modo que todos los alumnos tengan las mismas oportunidades. En otras palabras, hay que gestionar de alguna manera el conocimiento de la lengua en la clase de interpretación.

\subsection{Propuestas menos adecuadas para el aprendizaje y la enseñanza de la interpretación bi-activa}

Las siguientes propuestas didácticas nos parecen poco adecuadas por la falta de contexto:

- No anunciar ningún tema para la clase, es decir, suponer que los alumnos tienen un conocimiento latente de todos los temas posibles, y del léxico correspondiente. Nuestros alumnos son demasiado jóvenes como para tener conocimientos latentes sobre temas de política o economía. De este modo, le negamos al alumno la posibilidad de elaborar un conocimiento léxico sistemático en la lengua L2, L3, etcétera. Como no ha podido prepararse, el alumno se encuentra en una situación insegura y, por lo tanto, corre el riesgo de recurrir a muchos falsos amigos y estrategias de evitación. Tampoco le es posible desarrollar una estrategia de aprendizaje, porque en clase reina el azar. Es una situación que causa frustración y rechazo. Además, al final del curso, el profesor carece de criterios claros para evaluar al alumno, que vuelve a toparse con el dictado del azar.

- Anunciar un tema, pero sin proporcionar textos paralelos, obligando a los alumnos a que se preparen solos. Este modo de trabajar no conviene en una clase de interpretación para principiantes. Un principiante que tenga que prepararse por su cuenta no sabrá si su preparación estará a la altura de la clase. Es cierto que podrá trabajar de una forma más dirigida hacia un objetivo que en el primer caso, pero al igual que en el contexto anterior tendrá

Hildegard VERMEIREN Interpretar del neerlandés (L1) al español (L4) Propuestas pedagógicas 
que invertir mucha energía sin saber si su esfuerzo acabará siendo rentable. Si la preparación le sale bien, es por pura casualidad. Puede haber, además, grandes diferencias entre las actividades preparatorias llevadas a cabo por los alumnos, lo que complica la clase y obliga al docente a dar muchas explicaciones, entre otras, de carácter terminológico. También le será difícil evaluar los resultados de una manera justa, debido a la heterogeneidad de los mismos.

- Anunciar un tema facilitando un glosario bilingüe, pero sin textos. Este modo de trabajar tampoco conviene en una clase para principiantes, puesto que el glosario no aporta nada sobre el posible contenido de los ejercicios o charlas. Una vez más, es probable que el profesor se vea obligado a aclarar muchos de los términos y no pueda prestar la debida atención a los ejercicios de interpretación propiamente dichos. Para remediar este problema, conviene acompañar el glosario de una serie de textos. En una clase de nivel avanzado, donde los alumnos ya están más acostumbrados a la documentación previa, un glosario puede resultar interesante (siempre y cuando el alumno lo complete con los términos que vaya encontrando durante la preparación del encargo). El alumno debe familiarizarse, en primer lugar, con el contexto (el tema de la clase) para luego poder extraer el léxico. El método aquí descrito tampoco constituye un marco adecuado para evaluar a los estudiantes. Ningún profesor de interpretación puede pretender que sus alumnos den con la palabra justa cada vez que se presente la oportunidad de usar un término que aparezca en la lista.

- Hacer ejercicios en los que el alumno tiene que realizar traducciones palabra por palabra o frase por frase (de una lista) en la lengua extranjera, para averiguar si el alumno produce la palabra justa sin interferencias. Si el ejercicio está basado en una lista sin contexto, es poco probable que lleve a un conocimiento efectivo. Solo será útil si antes los estudiantes han trabajado con textos, y si es un ejercicio de control de unas pocas palabras, no de listas interminables.

Siguiendo a De Groot (2011, 89), creemos que estos métodos presentan debilidades porque tratan el léxico (y la gramática) fuera de contexto, es decir, no toman en consideración el elemento «conocimiento temático».

\subsection{Una propuesta basada en la realidad profesional}

Inspirándonos en Donovan (2005, 163), Gile (2009, 131) y Setton y Dawrant (2016a; 2016b), nuestra propuesta pedagógica se basa en el modo de trabajar del

Hildegard VERMEIREN Interpretar del neerlandés (L1) al español (L4) Propuestas pedagógicas 
intérprete profesional. Su forma de preparar los encargos revela un modo de aprender extremadamente intencional, relacionado con un contexto donde tendrá que poner a prueba sus conocimientos (De Groot 2011, 88). El intérprete explora este contexto a través de la intertextualidad, es decir, una serie de textos paralelos que le permiten relacionar conocimiento y lenguaje y tenerlos «disponibles» durante el encargo, o incluso a largo plazo.

En las clases de interpretación neerlandés-español en el Máster en Interpretación de la Universidad de Gante se aplican una serie de principios básicos. Los alumnos entran en un ciclo planificado que incluye la fase de preparación y dos horas semanales de clase (total: 90 minutos), y que detallamos a continuación.

- Definir un contenido preciso. En cada ciclo se trabaja en torno a un tema anunciado con una semana de antelación, lo cual permite una planificación cuidadosa. Algunos ejemplos de temas son: el sueño, el despilfarro de alimentos, las donaciones de sangre, el turismo en España/Bélgica, el cambio climático, la seguridad vial, el vapeo, los peligros de internet, la moda sostenible, etcétera. De esta manera, los alumnos pueden familiarizarse con una serie de temas (relacionados con la actualidad internacional y la realidad española) y con el léxico correspondiente. Como las semanas se organizan por temas, los estudiantes pueden memorizar mejor el contenido de los artículos así como el léxico. El contenido y el lenguaje son inseparables. Por lo general, un mayor conocimiento léxico contribuye a una mejor formulación, y hasta a un uso más eficaz de las estrategias de evitación.

- Delimitar el contenido. Para dos clases de hora y cuarto cada una, los alumnos reciben cinco textos, a saber, tres en español y dos en neerlandés, que juntos tienen una extensión de unas cuatro mil palabras. Se ofrece un mayor número de textos en la L4 para que los alumnos dispongan de más material para la producción en la lengua extranjera. Los textos no solo ofrecen contenido, sino también información léxica y gramatical. Los alumnos pueden aprovecharlos para encontrar significados equivalentes en ambas direcciones, reduciendo así las búsquedas en diccionarios. Se fijan en el léxico que se usa para hablar del tema y lo ordenan en un glosario, conscientes de que volverá a aparecer en los ejercicios de clase. De este modo, merece la pena hacer el esfuerzo de familiarizarse con el léxico y los aspectos gramaticales de los textos. Y, además, no hay necesidad de ampliar el glosario indefinidamente.

- Trabajar de modo circular. En clase, tanto el contenido de los textos como el léxico se utilizan en ejercicios de interpretación en ambas direcciones. Los alumnos no saben en qué dirección tendrán que hablar sobre qué tema. De esta manera, se le saca partido al tiempo invertido en el contenido y el lenguaje. Lógicamente, las clases también sirven para que los alumnos consoliden

Hildegard VERMEIREN Interpretar del neerlandés (L1) al español (L4) Propuestas pedagógicas 
el léxico y las estructuras gramaticales, lo cual redunda en beneficio de la disponibilidad léxica y gramatical. Es importante que durante la clase participe el mayor número posible de alumnos. Por eso fomentamos el trabajo por parejas o en grupos de tres. Como el objetivo es que los alumnos activen lo que han preparado o memorizado, no nos sirven las propuestas de Ko (1995), en cuyas clases participa un solo intérprete por turno. Tratamos de evitar los ejercicios en los que interpreta un solo estudiante mientras los demás permanecen callados. Esto supone poder disponer de un aula suficientemente grande para que puedan trabajar, por ejemplo, diez parejas.

- Durante la clase, averiguar si los alumnos aprovechan los materiales. Los ejercicios realizados en el aula sirven para consolidar los conocimientos de los alumnos. Eso implica que hayan hecho el esfuerzo de leer los textos y estudiar el léxico antes de acudir a clase. El profesor se pasea por el aula para escuchar cómo interpretan los alumnos y hacerles comentarios o correcciones. Si el profesor comprueba que hay mucha evitación y mala calidad de la traducción, es probable que el alumno no se haya preparado eficazmente y no esté aprovechando las clases. El profesor da feedback a los alumnos, en la misma clase o después de escuchar las grabaciones hechas en clase.

- En las charlas o discursos, reformular libremente los contenidos, no retomarlos literalmente. A pesar del carácter paralelo de los textos, las charlas y otros ejercicios del profesor deben ir incorporando elementos imprevisibles de forma paulatina, tal y como ocurre en la profesión. Dicha imprevisibilidad debe relacionarse más con el contenido que con el lenguaje porque, sobre todo al inicio de la formación, hay que evitar que el alumno se quede de repente bloqueado. Para que el alumno se sienta seguro, es importante que desarrolle un método de trabajo adecuado que le permita salir más o menos airoso de cualquier encargo de interpretación.

- Al final del curso basar el examen en los temas de clase. Si este método se aplica a lo largo de toda la asignatura, el alumno puede prepararse de modo eficaz a corto plazo para los exámenes, y a largo plazo para la profesión. Ahora bien, en el examen no se pide al alumno que repita literalmente un ejercicio hecho en clase, sino que trabaje con los temas tratados en el aula. Además, el método obedece a criterios más «justos». El profesor ofrece a todos sus alumnos las mismas posibilidades de éxito, ya que puede definir con claridad qué les va a exigir en el examen, por ejemplo en lo que se refiere a la calidad de la producción lingüística.

De esta manera, a través de sucesivos ciclos temáticos, el alumno incrementa su disponibilidad lingüística en general y léxica en particular. Al mismo tiempo, adquiere las técnicas de interpretación y las rutinas profesionales.

Hildegard VERMEIREN Interpretar del neerlandés (L1) al español (L4) Propuestas pedagógicas 
Además de los habituales ciclos semanales, la formación tiene una dimensión longitudinal que se desarrolla en tres fases. Al inicio, el profesor guía a los alumnos para que aprendan intencionalmente, pero poco a poco estos deben aprender a prepararse solos. También deben acostumbrarse a resolver situaciones imprevisibles. El Máster en Interpretación se ciñe a las fases 1 y 2 , en las que el profesor delimita el margen de movimiento y decide ampliarlo o no. Para el español como $L 4$, la fase 3 se produce más bien en la formación de intérpretes de conferencias. Lógicamente, en las lenguas donde el alumno alcanza el nivel C con mayor rapidez —como en el caso del francés y el inglés - es posible pasar a la fase 3 ya en el Máster.

Fase 1: Durante las seis primeras semanas del año de Máster, las clases se desarrollan con contenidos claramente delimitados, cada vez, mediante cinco textos paralelos que los alumnos deben preparar. Al inicio de la clase se comentan las dificultades (gramaticales o léxicas) de los textos, sin invertir tiempo en explicaciones que los alumnos pueden encontrar fácilmente en el diccionario o en los métodos de gramática de las clases de Grado. Los materiales y los ejercicios se van variando y mezclando (la interpretación al español puede ser una consecutiva, susurrada, traducción a vista, o interpretación bilateral). De este modo, la dificultad heurística y lingüística es menor y se puede trabajar la calidad. Los ejercicios retoman una y otra vez las palabras y las ideas de los textos. El objetivo es que los alumnos se den cuenta de que merece la pena leerlos, familiarizarse con el contenido y estudiar el léxico, porque, de ese modo, interpretar resulta más fácil.

Fase 2: A partir de la séptima semana y hasta el final del año de Máster, los temas preparados en las semanas anteriores se mezclan - de forma progresiva- con temas estrechamente relacionados pero no anunciados. De este modo, el alumno comprende que su preparación no solo le sirve para hablar de los temas de los textos proporcionados previamente. Se ve enfrentado a contenidos algo menos previsibles, y tiene que superar obstáculos poniendo en práctica la «disponibilidad lingüística" adquirida. Al tratarse de una $L 4$, conviene seguir trabajando de este modo durante algún tiempo.

Fase 3: (aplicable en interpretación de conferencias en L4) En esta fase también se trabaja en torno a un tema, pero los alumnos deben buscar los textos paralelos ellos mismos. Ya no se trata de trabajar en un circuito cerrado. La forma de trabajar sigue siendo circular, pero de modo menos explícito. Los alumnos eligen ellos mismos sus textos paralelos y prevén ellos mismos cuál va a ser el contenido de la charla o conferencia. A partir de sus lecturas, anticipan el contenido así como el lenguaje necesario para expresarlo (colocaciones, estructuras gramaticales, expresiones idiomáticas). Desarrollan las rutinas propias del intérprete mientras

Hildegard VERMEIREN Interpretar del neerlandés (L1) al español (L4) Propuestas pedagógicas 
se acercan paulatinamente al nivel C2 del MCERL. Su producción se vuelve más fluida, más correcta, más idiomática, es decir, de mejor calidad. Al final del Posgrado, los alumnos dominarán una serie de temas y su léxico específico en la L4. A este nivel, el profesor evaluará la dimensión lingüística de la producción oral, pero sus exigencias no podrán ser tan precisas como en el Máster, donde el léxico se deriva de un corpus de textos proporcionado por el profesor. Si los estudiantes se han documentado por su cuenta, no se les puede exigir que el léxico coincida plenamente con el que tenga en mente el profesor. Aun así, será fácil comprobar que algunos alumnos se han preparado más a fondo que otros. El profesor evaluará la calidad lingüística de la interpretación realizada por el alumno sin exigir que use una lista previamente pactada de términos o expresiones.

\section{CONCLUSIONES}

El intérprete que se gradúe en una facultad universitaria debe ser capaz de realizar una interpretación de calidad. El trabajo bi-activo resulta particularmente exigente a este respecto. La interpretación hacia una L4 como el español presenta un desafío incluso mayor que la interpretación hacia la L1. Aunque nuestros estudiantes de Máster han cursado tres años de Grado, y muchos incluso un semestre Erasmus en una universidad española, siguen produciendo errores como los que hemos recopilado en estas páginas. Cuantos más errores, mayor probabilidad de que el mensaje no se transmita fielmente. Por eso es importante que, al final del Máster en Interpretación, los estudiantes logren una producción fluida y correcta, algo que solo es posible si en clase nos esforzamos por incrementar la disponibilidad lingüística.

Lo que se ha escrito sobre la interpretación bi-activa en contextos bilaterales puede servir de marco, pero no aporta información concreta sobre cómo impartir una clase en la práctica. La experiencia acumulada a lo largo de una década como profesora del Máster en Interpretación de Gante ha dado lugar al método de enseñanza que se describe en este artículo para las clases de interpretación bi-activa neerlandésespañol. El método no es exclusivo de este par de lenguas, sino que se puede aplicar a cualquier otro par, siempre que se tenga en cuenta que los problemas translingüísticos no serán idénticos y que las lenguas estudiadas previamente varían de un contexto nacional a otro.

El método propuesto rechaza las soluciones elitistas que requieren que sean los propios estudiantes quienes se encarguen de buscar fórmulas para mejorar su nivel de L4 fuera de la universidad. Al contrario, pensamos que es preferible implementar un método que trate a todos los estudiantes por igual. No solo porque sea más justo, sino también porque, al final del curso, la evaluación se hace más sencilla.

Hildegard VERMEIREN Interpretar del neerlandés (L1) al español (L4) Propuestas pedagógicas 
Entre las competencias del intérprete destaca la facilidad de encontrar rápidamente equivalentes en el otro idioma, también en el idioma extranjero. Por ello, en las clases se debe prestar suficiente atención al incremento de la disponibilidad lingüística (léxica y gramatical) en la $L 4$, amén de otras competencias igualmente importantes, como la competencia intercultural o la competencia interactiva. Creemos que la mejor manera de obtener este incremento consiste en trabajar de manera cíclica y planificada, del mismo modo que cualquier encargo supone un ciclo planificado para el intérprete profesional: desarrollar conocimientos previos, tenerlos disponibles al momento y consolidarlos durante la interpretación. El profesional, de hecho, sigue ampliando sus conocimientos «disponibles" de esta manera. Por eso es conveniente que en clase se trabaje con un contenido determinado y que el mayor número posible de alumnos participe activamente en el cambio continuo de direccionalidad entre la L1 y L4. Este ir y venir fortalece la disponibilidad de todo lo aprendido, a saber, contenido y lenguaje. Además, a medio plazo, los conocimientos disponibles se volverán a activar durante el examen, que el alumno podrá encarar con mayor autoconfianza.

Está claro que no todos los alumnos tienen el mismo nivel de disciplina ni las mismas aptitudes para estudiar o sortear dificultades, y no todos serán intérpretes durante su vida profesional. Pero al menos conocerán el método básico que le permite al intérprete profesional lograr una interpretación de calidad hacia su L4.

\section{REFERENCIAS BIBLIOGRÁFICAS}

Collados Aís, Angela y María Manuela FERNÁNDEZ SÁNCHEZ. 2001. Manual de interpretación bilateral. Granada: Comares.

CONSEJO DE EUROPA. 2002. Marco Común Europeo de Referencia para las Lenguas. Aprendizaje, Enseñanza, Evaluación. Madrid: Ministerio de Cultura, Educación y Deporte. Fecha de acceso 25 de octubre de 2016. http://cvc.cervantes.es/ensenanza/biblioteca ele/marco/ cvc mer.pdf

DE BRUYNE, Jacques y Lieve BEHIELS. 2014. Nieuwe Spaanse Grammatica. Gent: Academia Press.

DE GROOT, Annette B. 2011. Language and Cognition in Bilinguals and Multilinguals. New York/ Hove: Psychology Press.

DONOVAN, Claire. 2005. «Teaching Simultaneous Interpretation into B. A Challenge for Responsible Interpreter Training”. En Directionality in Interpreting. The 'Retour' or the Native?, ed. por Rita Godijns y Michael Hinderdael. Gent: Communication and Cognition 38 (1/2): 147-165.

DE SWAAN, Abram. 2001. The Words of the World: The Global Language System. Cambridge: Polity Press.

DG COMMUNICATION. 2012. Les Européens et leurs langues. Eurobaromètre spécial 386. Brussel/Bruxelles: Commission Européenne.

Hildegard VERMEIREN Interpretar del neerlandés (L1) al español (L4) Propuestas pedagógicas 
GILE, Daniel. 2005. «Directionality in Conference Interpreting. A Cognitive View». En Directionality in Interpreting. The 'Retour' or the Native?, ed. por Rita Godijns y Michael Hinderdael. Gent: Communication and Cognition 38 (1/2): 147-166.

GILE, Daniel. 2009. Basic Concepts and Models for Interpreter and Translator Training. Amsterdam/Philadelphia: John Benjamins (Benjamins Translation Library, 8).

GREEN, David W. 1998. "Mental Control of the Bilingual Lexico-Semantic System». Bilingualism: Language and Cognition 1 (2): 76-81.

GUIJARRO-FUENTES, Pedro. 2012. "The Acquisition of Interpretable Features in L2 Spanish: Personal a». Bilingualism: Language and Cognition 15 (4): 701-720.

HATIM, Basil. 1997. Communication across Cultures. Translation Theory and Contrastive Text Linguistics. Exeter: University of Exeter Press (Exeter Linguistic Studies).

HUFEISEN, Britta y Gerhard NEUNER. 2004. The Plurilingualism Project: Tertiary Language Learning - German after English. Strasbourg: Council of Europe Publishing. Fecha de acceso 19 de octubre de 2016. http://archive.ecml.at/documents/pub112E2004HufeisenNeuner.pdf

ILIESCU GHEORGHIU, Catalina. 2004. Introducción a la interpretación consecutiva. Alicante: Universidad de Alicante.

JONES, Roderick. 1998. Conference Interpreting Explained. Manchester: St Jerome.

KALINA, Sylvia. 2005. «Quality in the Interpreting Process: What Can Be Measured and How?». En Directionality in Interpreting. The 'Retour' or the Native?, ed. por Rita Godijns y Michael Hinderdael. Gent: Communication and Cognition 38 (1/2): 147-166.

KO, Leong. 1995. «Teaching Dialogue Interpreting». En Teaching Translation and Interpreting 3, ed. por Cay Dollerup y Vibeke Appel. Amsterdam/Philadelphia: John Benjamins, 119-128.

LINN, Stella y Miel SLAGER. 2007. Vertalen uit het Spaans. Bussum: Coutinho.

LUJÁN Castro, José. 2002. La enseñanza del español en Bélgica. Brussel/Bruxelles: Consejería de Educación.

PADILLA, Presentación. 2005. «Cognitive Implications of the English-Spanish Direction for the Quality and the Training of Simultaneous Interpreting". En Directionality in Interpreting. The 'Retour' or the Native?, ed. por Rita Godijns y Michael Hinderdael. Gent: Communication and Cognition 38 (1/2): 47-62.

ORTEGA, Lourdes. 2009. Understanding Second Language Acquisition. London: Hodder.

PÖCHHACKER, Franz. 2004. Introducing Interpreting Studies. London: Routledge.

REAL ACADEMIA ESPAÑOLA. 2009. Nueva gramática de la lengua española. Madrid: Espasa Libros.

SELESKOVITCH, Danica y Marianne Lederer. 1989. Pédagogie raisonnée de l'interprétation. Paris: Minard.

SELIGER, Herbert W. y Elana SHOHAMY. 1989. Second Language Research Methods. Oxford: Oxford University Press.

SOCIAAL-ECONOMISCHE RAAD VOOR VLAANDEREN (SERV). 2008. Competentieprofiel Sociaal Tolken. Brussel/Bruxelles: SERV. Fecha de acceso 24 de octubre de 2016. http://www. kruispuntmi.be/sites/default/files/bestanden/documenten/beroepscompetentieprofiel sociaal tolk.pdf

SETtON, Robin y Andrew DRAWANT. 2016a. Conference Interpreting. A trainer's Guide. Amsterdam/Philadelphia: John Benjamins.

Hildegard VERMEIREN Interpretar del neerlandés (L1) al español (L4) Propuestas pedagógicas 
SETTON, Robin y Andrew DRAWANT. 2016b. Conference Interpreting. A Complete Course. Amsterdam/Philadelphia: John Benjamins.

VAN DER SIJS, Nicoline. 2009. «Import en export van woorden. Tijd voor een balans». Tijdschrift voor Nederlandse Taal- en Letterkunde 125: 194-200. 